\title{
Vitamin D enhances reactive oxygen intermediates production in phagocytic cells in term and preterm infants
}

\author{
Chike Onwuneme ${ }^{1,2,3}$, Alfonso Blanco ${ }^{2}$, Amanda O'Neill ${ }^{2}$, Bill Watson ${ }^{2}$ and Eleanor J. Molloy ${ }^{1,45,5,6,7}$
}

BACKGROUND: Newborn infants are endotoxin tolerant which may be responsible for their increased susceptibility to bacterial sepsis. Vitamin D has an immunomodulatory effect and newborn infants are at risk of vitamin D deficiency. We examined the in vitro effect of 1, 25-dihydroxyvitamin D $(1,25 \mathrm{OHD})$ on whole blood phagocytic toll-like receptor 4 (TLR4), CD11b, and reactive oxygen intermediates (ROIs) in newborn infants during sepsis.

METHODS: Whole blood from preterm infants $<32$-wk gestation, control term neonates, and adults were sampled for phagocytic expression of ROI, TLR4, CD 11 b in response to lipopolysaccharide (LPS), and 1,250HD using flow cytometer.

RESULTS: ROI production from newborn phagocytes incubated with LPS alone was decreased. Pretreatment with $1,25 \mathrm{OHD}$ demonstrated increased $(P=0.001)$ phagocytic ROI production in newborns but not in adults. 1,250HD did not have any effect on TLR4 and CD11b in both newborns and adults. Pretreatment with ROI inhibitors (apocynin (APO) and diphenyleneiodonium), phosphoinositide 3-kinase (PI3K) inhibitor, and p38 inhibitor blocked neutrophil ROI production. CONCLUSION: Neonatal phagocytic cells had diminished $\mathrm{ROI}$ production in the presence of LPS, however, pretreatment with $1,25 \mathrm{OHD}$ reversed this hyporesponsiveness. This action by $1,25 \mathrm{OHD}$ was mediated by activation of nicotinamide adenine dinucleotide phosphate oxidase system through PI3K signaling enzymes.

Ni eonates can be immune tolerant in the presence of infection, and this state of immune hyporesponsiveness potentially increases neonatal vulnerability to infection $(1,2)$. Endotoxin tolerance is a reduced responsiveness to a bacterial lipopolysaccharide (LPS) challenge following a first encounter with endotoxin. The respiratory burst is an essential mechanism by which neutrophils and monocytes kill invading micro-organisms (3). Antimicrobial activity by newborn neutrophils is decreased (4) due to an altered nicotinamide adenine dinucleotide phosphate (NADPH) oxidase system which is even further decreased in preterm infants (5). Although there is increased superoxide anion $\left(\mathrm{O}_{2}^{-}\right)$production in cord blood compared with adults, the generation of hydroxyl radical (.OH) is relatively decreased (6). This defect contrasts to adult neutrophils with increased respiratory burst in response to bacterial pathogens (7).

The processes of neutrophil adhesion and diapedesis are mediated by the CD11b subunit adhesion molecule of macrophage-1 antigen (Mac-1) (4). Impairment of neutrophil adherence, chemotaxis, and migration in neonates increases their susceptibility to infection in the first month of life (8). Tolllike receptor 4 (TLR4) plays an important role in detecting microbial infection and triggering antimicrobial host defense responses and endotoxin signaling $(9,10)$. Neonatal neutrophils displayed increased TLR4 expression following heat shock and LPS, in contrast to adults (11). Immunomodulation may protect neonates with sepsis. 1,25-dihydroxyvitamin D $(1,25 \mathrm{OHD})$ exerts biological effects in isolated innate immune cells, but its effect in whole blood neutrophils and monocytes TLR4, reactive oxygen intermediate (ROI), and CD11b is unclear. We hypothesized that vitamin D may have a biological effect in whole blood innate immune cells of neonates. We aimed to examine the in vitro effect of $1,25 \mathrm{OHD}$ on whole blood neutrophils and monocytes TLR4, CD11b, and ROI from preterm and term infants.

\section{RESULTS}

\section{Patient Characteristics}

There were 16 healthy adult controls, 12 healthy term neonatal controls, and 17 preterm infants $<32$-wk gestation in this study. The adult controls had a mean \pm SD age of $37 \pm 6 y$, with seven males and nine females. The healthy term neonatal controls had a mean \pm SD gestational age of $40 \pm 1 \mathrm{wk}$, and there were eight males and four females. Preterm infants had a mean gestational age of $30 \pm 2 \mathrm{wk}$, of which eight were males and nine were females. Mean \pm SD serum vitamin D status was available for 14 of the 16 healthy adult controls $(41.5 \pm 30 \mathrm{nmol} / \mathrm{l})$, 11 of the 12 healthy term neonatal controls $(41.2 \pm 27 \mathrm{nmol} / \mathrm{l})$,

\footnotetext{
'Department of Neonatology, National Maternity Hospital, Dublin, Ireland; ${ }^{2}$ University College Dublin School of Medicine and Medical Science, Conway Institute for Biomolecular and Biomedical Science, University College Dublin, Dublin, Ireland; ${ }^{3}$ Department of Paediatrics, Children's University Hospital, Dublin, Ireland; ${ }^{4}$ Department of Paediatrics, Royal College of Surgeons, Dublin, Ireland; ${ }^{5}$ Department of Neonatology, Our Lady's Children's Hospital, Dublin, Ireland; ${ }^{6}$ Department of Paediatrics, Trinity College Dublin, National Children's Hospital, Dublin, Ireland; ${ }^{7}$ Department of Neonatology, Coombe Women's and Infants University Hospital, Dublin, Ireland. Correspondence: Chike Onwuneme (chike.onwuneme@gmail.com)

Received 3 February 2015; accepted 30 September 2015; advance online publication 20 January 2016. doi:10.1038/pr.2015.268
} 
and from all the preterm infants $(43.2 \pm 37 \mathrm{nmol} / \mathrm{l})$. Vitamin $\mathrm{D}$ status on the average was below $50 \mathrm{nmol} / \mathrm{l}$ in all the groups. However, we noticed a positive correlation between vitamin D status and ROI production after pretreatment with $1,25 \mathrm{OHD}$ in term infants $\left(r^{2}=0.48\right)$.



Pretreatment of Whole Blood With 1,250HD and Effects on TLR4, CD11b, and ROI

Whole blood were pretreated and incubated from healthy adult controls $(n=10)$, term neonatal controls $(n=10)$, and preterm infants $(n=10)$ with $1,25 \mathrm{OHD}$ for $1 \mathrm{~h}$ before addition

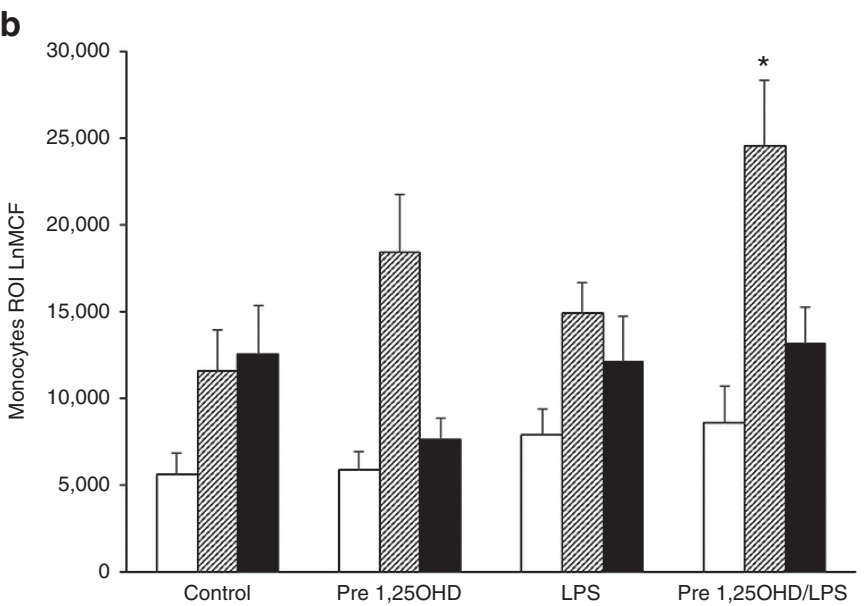

Figure 1. Pretreatment with 1, 25-dihydroxyvitamin D (1,25OHD) and reactive oxygen intermediate (ROI) production in neonates. Whole blood (a) neutrophils and (b) monocytes ROI production after pretreatment with 1,25OHD and incubated with lipopolysaccharide (LPS), from healthy adults (white bar, $n=10$ ), preterm infants $<32$-wk gestation within $24 \mathrm{~h}$ of delivery (black bar, $n=10$ ), and umbilical cord samples from term neonates (hatched bar, $n=10$ ) after vaginal delivery. Pretreatment with 1,25OHD demonstrated a significant increase in ROI production for both preterm and term neonates. ${ }^{*}$ Term control vs. term 1,25OHD: $P=0.01$. ${ }^{*}$ Term LPS vs. term 1,25OHD/LPS: $P=0.001$; ${ }^{+}$Preterm infants' LPS vs. preterm infants' $1,25 \mathrm{OHD} / \mathrm{LPS}: P=0.001$. Results were expressed as the Ln mean channel fluorescence (LnMCF) and error bars displayed as SEM.

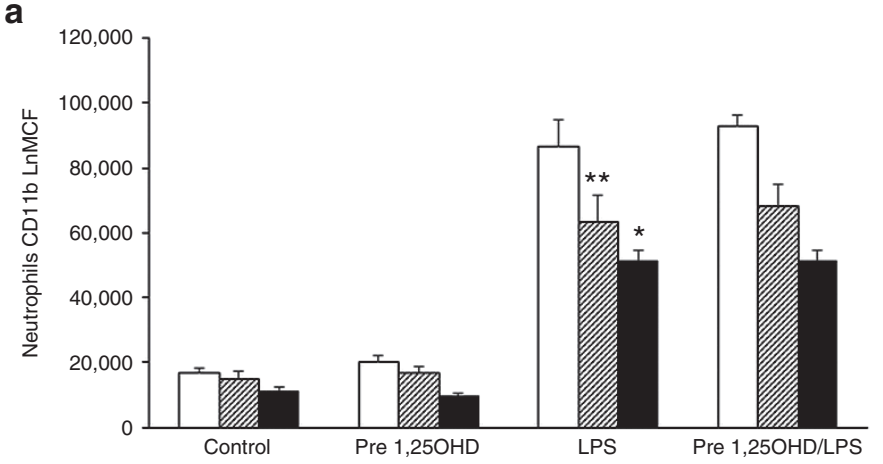

b

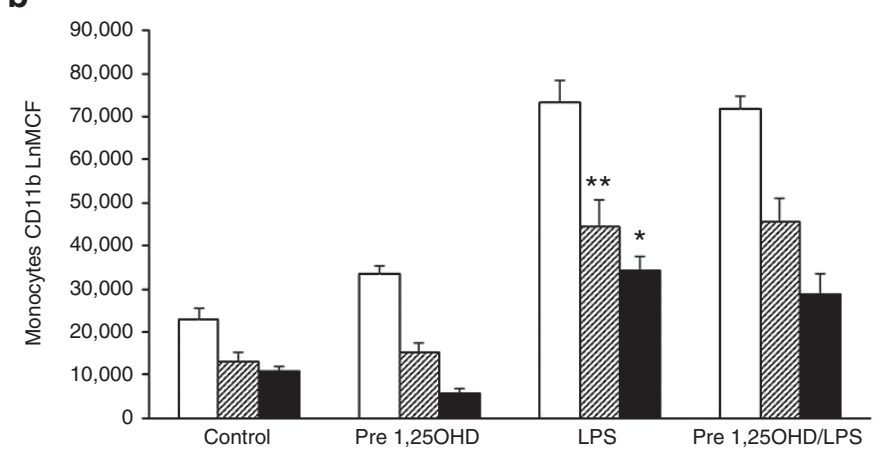

C

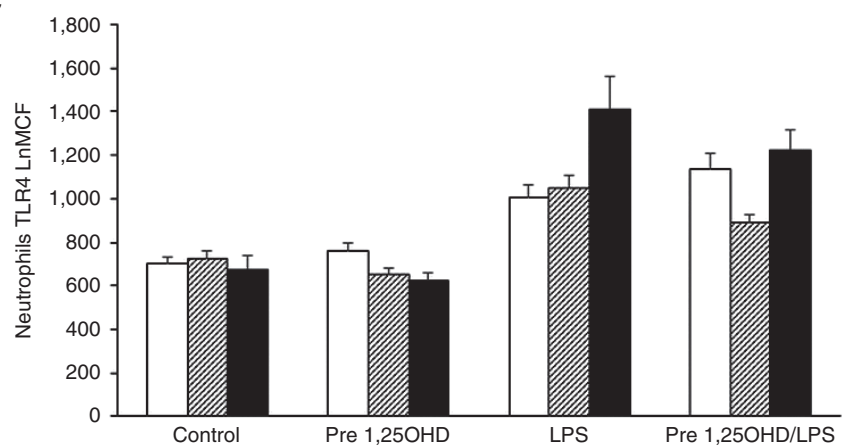

d



Figure 2. Pretreatment with 1, 25-dihydroxyvitamin D (1,25OHD) and CD11b/TLR4 expression. Whole blood (a) CD11b neutrophils and (b) monocytes and (c) toll-like receptor 4 (TLR4) neutrophils and (d) monocytes expression after pretreatment with 1,25OHD and incubated with lipopolysaccharide (LPS), from healthy adults (white bar, $n=10$ ), preterm infants $<32$-wk gestation within $24 \mathrm{~h}$ of delivery (black bar, $n=10$ ), and umbilical cord samples from term neonates (hatched bar, $n=10$ ) after vaginal delivery. Preterm infants' and term controls' CD11b expression in the presence of LPS is lower than in adult controls $(* P<0.0001)$ and $(* * P=0.05)$, respectively. Pretreatment with $1,25 \mathrm{OHD}$ had no effect on CD11b and TLR4 expression in all groups. Results were expressed as Ln mean channel fluorescence (LnMCF) and error bars displayed as SEM. 
of LPS with no significant difference in both neutrophil and monocyte ROI baseline expression between preterm infants and both control groups (Figure 1). ROI production from preterm and term neonatal neutrophils incubated with LPS alone was diminished and almost similar to baseline (control). However pretreatment with 1,25OHD before adding LPS demonstrated an increase $(P=0.001)$ in ROI production in neutrophils for both preterm and term neonates (Figure 1a). Pretreatment with $1,25 \mathrm{OHD}$ also increased ROI production in term control infants independent of LPS. Pretreatment with $1,25 \mathrm{OHD}$ before adding LPS also demonstrated a significant increase $(P=0.04)$ in ROI production in monocytes for term neonatal controls (Figure 1b).

Neutrophil CD11b baseline expression and LPS upregulation showed no significant difference between preterm infants and both control groups (Figure 2a). CD11b expression of preterm infants and term neonatal controls in the presence of LPS was lower than in adult controls $(P<0.0001$ and $P=0.05$, respectively). Similarly, monocytes CD11b expression of preterm infants and term neonatal controls in the presence of LPS was lower than in adult controls $(P<0.0001$ and $P=0.002$, respectively; Figure 2b). Pretreatment with 1,25OHD had no effect on neutrophil or monocyte $\mathrm{CD} 1 \mathrm{~b}$ expression in both preterm infants and controls. There was no difference in both neutrophil and monocyte TLR4 baseline and LPS-induced expression between preterm infants and both control groups (Figure 2c,d). Neutrophil TLR4 expression of preterm patients in the presence of LPS was increased compared with adult LPS TLR4 expression $(P=0.02$; Figure $2 c)$. Pretreatment with $1,25 \mathrm{OHD}$ had no effect on neutrophil and monocyte TLR4 expression in both preterm infants and controls. Similar expressions of CD11b, TLR4, and ROI as with pretreatment with 1,25OHD were noted in both neutrophils and monocytes, however, simultaneous incubation with 1,25OHD had no effect on neutrophil and monocyte TLR4, CD11b, and ROI in all groups (results not shown).

\section{Pretreatment With Specific NADPH Oxidase Inhibitor APO and Nonspecific NADPH Oxidase Inhibitor DPI Blocks 1,250HD- Enhanced Neutrophil ROI Production}

We assessed the contribution of NADPH oxidase to enhanced neutrophil ROI production observed in 1,25OHD pretreated term neonatal whole blood. Pretreatment with 1,25OHD before adding LPS demonstrated a significant increase $(P=0.0001)$ in neutrophil ROI production, but pretreatment with specific (apocynin (APO)) and nonspecific (diphenyleneiodonium (DPI)) NADPH oxidase inhibitors blocked this effect in a dose-dependent manner (Figure 3a,b).

\section{PI3K Inhibitor LY 294002 and p38 Inhibitor SB 203580 Suppressed 1,25OHD-Enhanced Neutrophil ROI Production} Kinases participate in NADPH oxidase activation including protein Kinase C (PKC), p38 mitogen-activated protein kinase (p38 MAPK), extracellular signal-regulated kinase (ERK), and phosphoinositide 3-kinase (PI3K). PI3K regulates 1,25OHD ROI production in human monocytic cell lines $(12,13)$. PI3K inhibitor LY 294002 and P38 inhibitor SB 203580 suppressed $1,25 \mathrm{OHD}$ augmented LPS ROI production even at $1 \mu \mathrm{M}$ (Figure 4a,b). However, the ERK inhibitor, PD 98059, had an
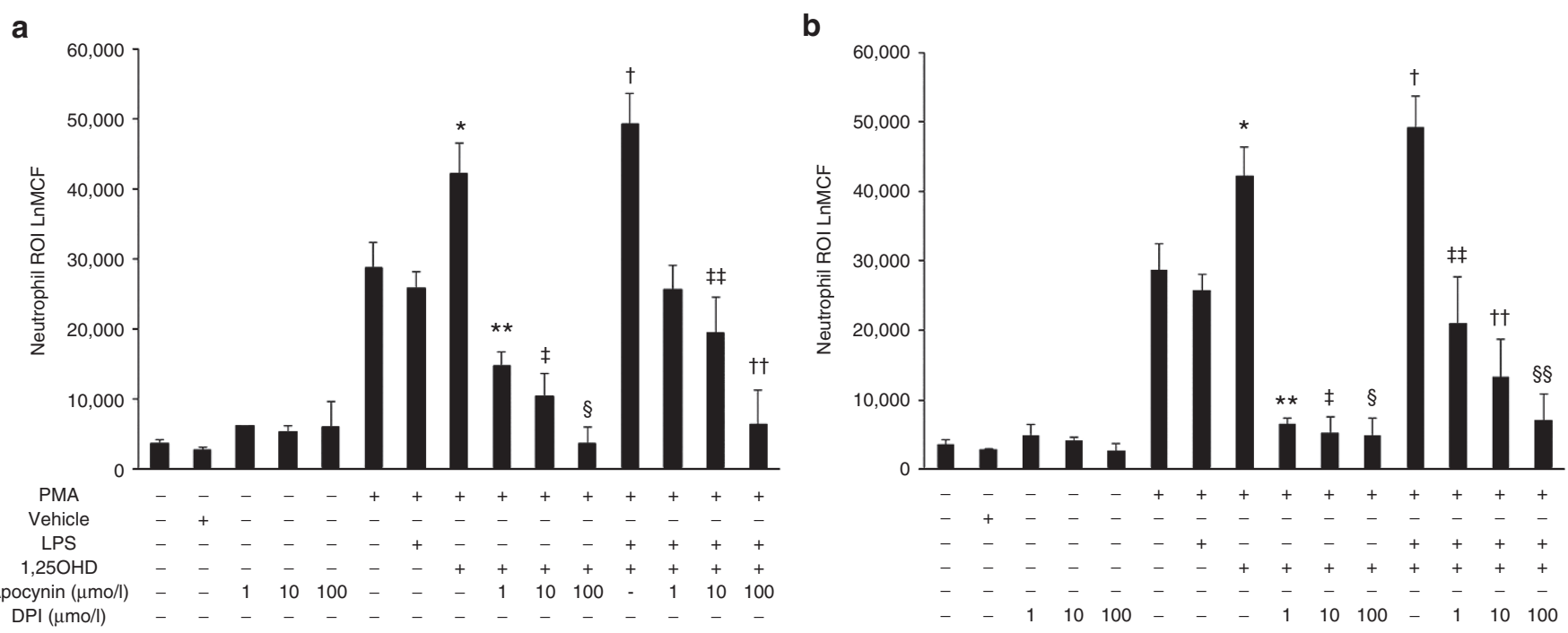

Figure 3. Pretreatment with specific nicotinamide adenine dinucleotide phosphate (NADPH) oxidase inhibitors, apocynin (APO), and nonspecific NADPH oxidase inhibitor, diphenyleneiodonium (DPI), blocks 1, 25-dihydroxyvitamin D (1,25OHD)-enhanced neutrophil reactive oxygen intermediate (ROI) production. Whole blood from term infants $(n=3)$ were pretreated and incubated with specific NADPH oxidase inhibitors, (a) APO and nonspecific NADPH oxidase inhibitor, (b) DPI for 30 min prior to experiments. Pretreatment with both inhibitors blocks 1,25OHD-enhanced ROI production in a dose-dependent manner. Results were expressed as Ln mean channel fluorescence (LnMCF) and error bars displayed as SEM. a: *1,25OHD+PMA vs. PMA: $P=0.03 ;{ }^{* *} 1,25 \mathrm{OHD}+\mathrm{PMA}+\mathrm{APO} 1 \mu \mathrm{M}$ vs. $1,25 \mathrm{OHD}+\mathrm{PMA}: P=0.02 ;{ }^{\ddagger} 1,25 \mathrm{OHD}+\mathrm{PMA}+\mathrm{APO} 10 \mu \mathrm{M}$ vs. $1,25 \mathrm{OHD}+\mathrm{PMA}: P=0.002 ;{ }^{\S} 1,25 \mathrm{OHD}+\mathrm{PMA}+\mathrm{APO}$

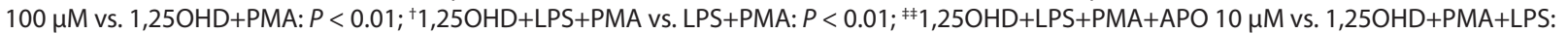
$P<0.01 ;{ }^{+1} 1,25 \mathrm{OHD}+\mathrm{LPS}+\mathrm{PMA}+\mathrm{APO} 100 \mu \mathrm{M}$ vs. $1,25 \mathrm{OHD}+\mathrm{PMA}+\mathrm{LPS}: P<0.01 . \mathbf{b}:{ }^{*} 1,25 \mathrm{OHD} 3+\mathrm{PMA}$ vs. PMA: $P=0.03 ;{ }^{* *} 1,25 \mathrm{OHD}+\mathrm{PMA}+\mathrm{DPI} 1 \mu \mathrm{M}$ vs. $1,25 \mathrm{OHD}+\mathrm{PMA}: P=0.004 ;{ }^{\ddagger} 1,25 \mathrm{OHD}+\mathrm{PMA}+\mathrm{DPI} 10 \mu \mathrm{M}$ vs. $1,25 \mathrm{OHD}+\mathrm{PMA}: P<0.01 ;{ }^{\S} 1,25 \mathrm{OHD}+\mathrm{PMA}+\mathrm{DPI} 100 \mu \mathrm{M}$ vs. $1,25 \mathrm{OHD}+\mathrm{PMA}: P<0.01$;

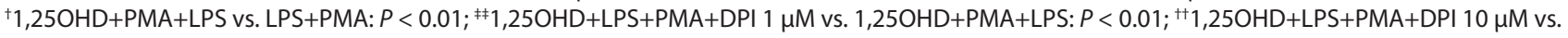

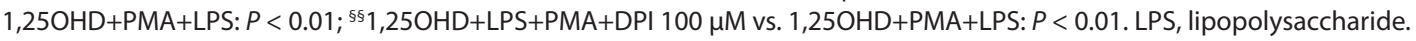


a

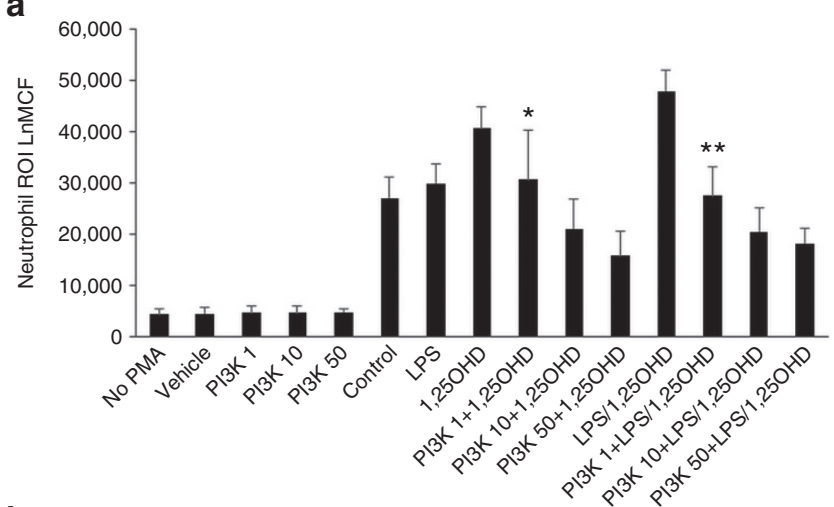

b



C



d

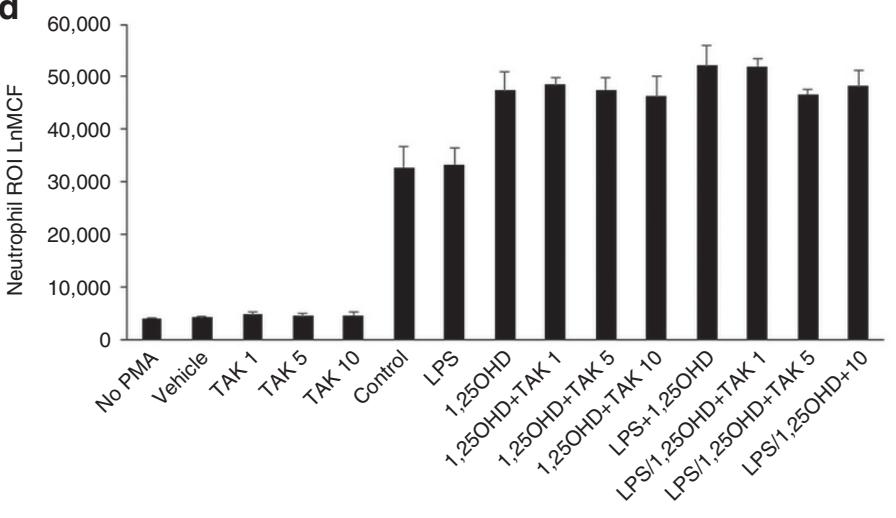

Figure 4. Phosphoinositide 3-kinase (PI3K) inhibitor LY 294002 and p38 inhibitor SB 203580 suppressed 1, 25-dihydroxyvitamin D (1,25OHD)-enhanced neutrophil reactive oxygen intermediate (ROI) production. Whole blood from term infants $(n=3)$ were pretreated and incubated with the inhibitors for 30 min prior to experiments. (a) PI.3K inhibitor LY 294002 suppressed 1,25OHD augmented neutrophil ROI production. ${ }^{*} 1,25 O H D+P I 3 K ~ 10 \mu M$


no effect on 1,25OHD-enhanced ROI production except at a high concentration. ${ }^{*} 1,25 \mathrm{OHD}+\mathrm{ERK} 50 \mu \mathrm{M}$ vs. 1,25OHD: $P=0.05 ; * * 1,25 \mathrm{OHD}+\mathrm{LPS}+\mathrm{PI} 3 \mathrm{~K}$ $10 \mu$ M vs. 1,25OHD+LPS: $P=0.01$. (c) P38 inhibitor SB 203580 suppressed 1,25OHD augmented ROI production at lowest concentration of $1 \mu M$. *1,25OHD+LPS+p38 $1 \mu$ M vs. 1,25OHD+LPS: $P=0.02$. (d) Specific toll-like receptor 4 blocker, TAK-242, had no effect on 1,25OHD ROI production. Results were expressed as Ln mean channel fluorescence (LnMCF) and error bars displayed as SEM. LPS, lipopolysaccharide.

effect only at a very high concentration of $100 \mu \mathrm{M}$ (Figure $4 \mathrm{c}$ ). Having established that blocking downstream PI3K and p38 in the LPS/TLR4 pathway suppressed 1,25OHD augmented increase in term infants' neutrophil ROI production, we found that blocking TLR4 had no effect on 1,25OHD ROI production (Figure $4 \mathrm{~d}$ ) implying that $1,25 \mathrm{OHD}$ is not dependent on TLR4 receptor for its effect on ROI production.

\section{1,250HD Effect on Phosphorylation of Akt, p38, and ERK Signaling Proteins}

Expression of phosphorylated PI3K, p38, and ERK (Figure 5) shown as Ln mean channel fluorescence was observed in both untreated and stimulated samples. There was a significant difference in the expression of phosphorylated p38 $(P=0.0001)$ and ERK $(P=0.0006)$ between untreated and samples stimulated with phorbol myristate acetate (PMA) (Figure 5a,b). $1,25 \mathrm{OHD}$ did not show any effect on the expression of phosphorylated Akt, p38, or ERK.

\section{DISCUSSION}

We have demonstrated that ROI production from preterm and term neonatal neutrophils in whole blood incubated with LPS alone was unchanged and almost similar to baseline. However, pretreatment of both preterm and term neonatal whole blood with $1,25 \mathrm{OHD}$ before incubation with LPS increased neutrophil and monocyte production of ROI. This effect was higher in term compared with preterm infants. Our study showed a positive correlation between vitamin $\mathrm{D}$ status and ROI production in term infants with maximal effect at a vitamin D status of approximately $65 \mathrm{nmol} / \mathrm{l}$ beyond which no added effect was noticed. Yang et al. (14) reported that increased ROI production also inhibits granulopoiesis under endotoxin stimulation. Therefore, increased ROI production may combat infection but has a risk of increased tissue damage and decreased granulopoiesis. Increased production of ROI in term neonatal neutrophils was decreased by pretreatment with increasing concentrations of specific NADPH oxidase inhibitor APO and nonspecific inhibitor DPI, thus confirming 1,25OHD action was mediated by the NADPH oxidase. There was inhibition of ROI production when only $1,25 \mathrm{OHD} 3$ was added to samples containing APO and DPI compared with when both 1,25OHD and LPS were added. This finding indicates that NADPH oxidases are the major sources of ROI in 1,25OHD-augmented ROI production, but NADPH oxidases may not be the only 


\section{Articles | Onwuneme et al.}

a

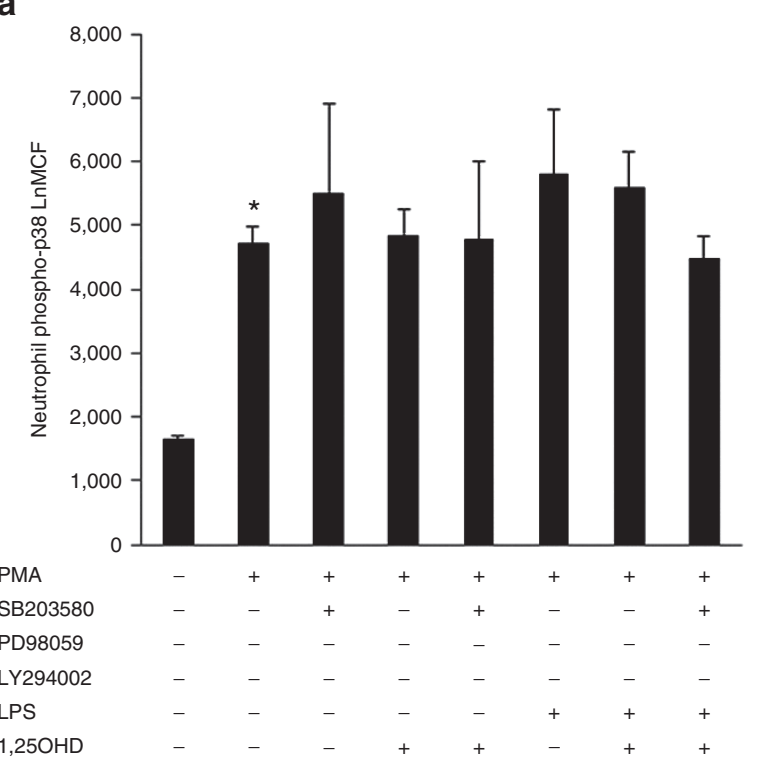

b

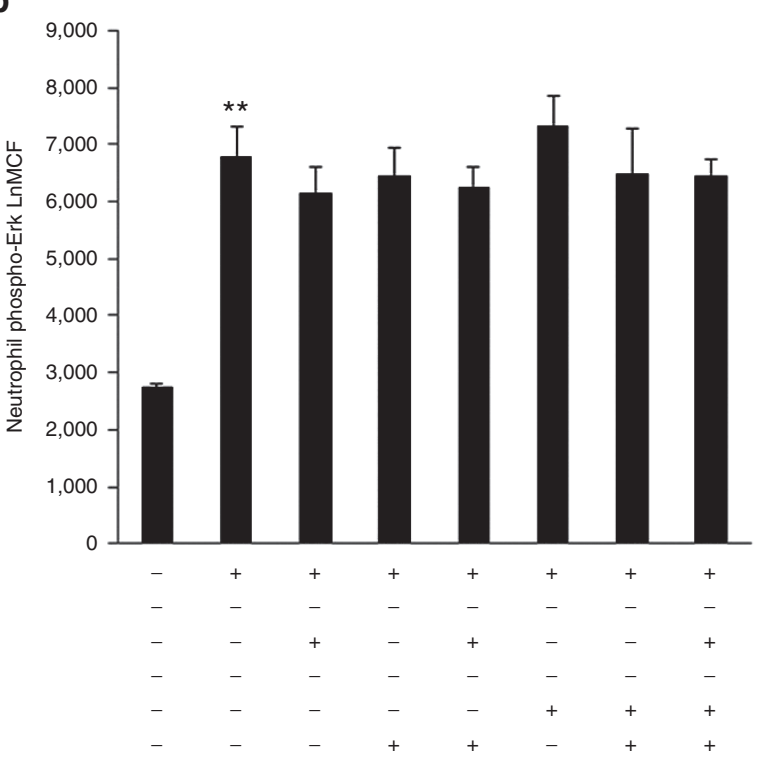



Figure 5. Effect of 1,25-dihydroxyvitamin $\mathrm{D}(1,25 \mathrm{OHD})$ on phosphorylation of Akt, $\mathrm{p} 38$, and extracellular signal-regulated kinase (ERK) signaling proteins. Whole blood from healthy term infants $(n=5)$ was incubated with $(\mathbf{a}) \pm$ p38 mitogen-activated protein kinase (p38 MAPK) inhibitor SB 203580 , *PMA vs. untreated: $P \leq 0.01$; (b) ERK inhibitor PD 98059, **PMA vs. untreated: $P \leq 0.01$; and (c) phosphoinositide 3-kinase inhibitor LY 294002 and was pretreated with $\pm 1,25 \mathrm{OHD}$ before incubating with lipopolysaccharide (LPS). Results were expressed as Ln mean channel fluorescence (LnMCF) and error bars displayed as SEM.

source of ROI in LPS-treated neutrophils. We demonstrated that this $1,25 \mathrm{OHD}$ action in term neonatal whole blood neutrophils was effected through p38 and PI3K enzymes. PI3K inhibitor LY 294002 and p38 inhibitor SB 203580 suppressed $1,25 \mathrm{OHD}$-augmented LPS ROI production even at $1 \mu \mathrm{M}$.

There was a difference in the expression of phosphorylated p38 and ERK between untreated and stimulated samples. However, this difference was not noted in Akt signaling. One possible explanation of this is that freshly prepared cells show Akt phosphorylation, which is PI3K dependent (15) and could mask any difference between untreated and stimulated cells noticed in our results for Akt. Our results showed a trend of increased Akt phosphorylation in 1,25OHD/LPS-treated samples and inhibition of PI.3K showed a trend toward inhibition of Akt phosphorylation though these were not statistically significant. 1,25OHD did not show any effect on the expression of phosphorylated p38 or ERK. Blocking TLR4 had no effect on $1,25 \mathrm{OHD}$ ROI production, implying that $1,25 \mathrm{OHD}$ is not dependent on TLR4 receptor for its effect on ROI production similar to PMA action on ROI production which is receptor independent. From our results, 1,25OHD either directly activates PI.3K enzymes or activates other enzymes downstream of TLR4, which in turn ultimately activates PI.3K enzymes and ultimately activates ROI production through NADPH oxidase. 
We also showed that simultaneous addition of $1,25 \mathrm{OHD}$ with LPS had no effect in either term or preterm neonatal whole blood neutrophils and monocytes. These findings may imply that giving vitamin $\mathrm{D}$ to newborn infants at time of sepsis will not have any effect in bactericidal action through ROI production, but having sufficient vitamin D before sepsis may induce antibacterial action via this mechanism. Our results showed no significant difference in both baseline and LPS-induced whole blood neutrophil ROI production between preterm and term infants. Björkqvist et al. (5) showed a similar baseline neutrophil ROI in term and preterm neonates. However, term infants have significantly increased oxidative burst in response to coagulase-negative staphylococci in vitro compared with preterm infants.

Previous studies in human monocytic cell line had shown that $1,25 \mathrm{OHD}$ induced generation of ROIs and was antimycobacterial $(12,13)$. This effect was shown to be regulated by PI3K and mediated by NADPH oxidase system, although the effects of p38 or ERK effector enzymes were not assessed. Hmama et al. (12) postulated that 1,25OHD treatment induced the formation of a vitamin $\mathrm{D}$ receptor-PI3K complex representing a novel nongenomic signaling pathway activated by $1,25 \mathrm{OHD}$.

We have shown similar baseline whole blood neutrophil CD11b expression in preterm neonates compared with adults in keeping with other studies $(16,17)$. We found that whole blood neutrophils and monocytes CD11b expression was significantly increased in response to LPS in each group compared with baseline. CD11b expression of preterm infants and term neonatal controls in the presence of LPS was significantly lower than in adult controls, and this was more obvious in preterm patients. We did not show any effect of $1,25 \mathrm{OHD}$ on neutrophil and monocyte CD11b expression in both preterm infants and controls, although increased CD11b expression in THP- 1 cells has been shown in response to $1,25 \mathrm{OHD}$ (12). This could be explained by the fact that cell lines are not representatives of human cells and may respond differently. THP-1 cells are widely applied to mimic monocytes in cell culture models but differ in functional responses comparable with whole blood. Schildberger et al. (18) demonstrated that THP-1 cells differ from whole blood with respect to cytokine release after stimulation with LPS. Watson et al. (19) demonstrated that isolation procedures prime both receptor expression and oxidase function in isolated cells compared with whole blood. Thus, the use of whole blood, as in our study, had an advantage of less interference with cell function and serum factors.

We showed no significant difference in whole blood neutrophil and monocyte TLR4 baseline expression between preterm infants and both control groups. LPS-induced whole blood neutrophil TLR4 expression was higher in preterm neonates compared with control groups though not statistically significant, and pretreatment with 1,250HD or simultaneous addition in the presence of LPS did not show an effect. Sadeghi et al. (20) had previously demonstrated downregulation of TLR4 expression after 1,25OHD was incubated for $12-72 \mathrm{~h}$ in human cultured monocytic cell lines which is different to whole blood neonatal samples used in our experiments.
In conclusion, we have demonstrated that newborn preterm infants were hyporesponsive to LPS with decreased neutrophil ROI production and CD11b expression compared with controls which recovered upon pretreatment with $1,25 \mathrm{OHD}$ with regard to ROI production. 1,25OHD may enhance neonatal whole blood neutrophil ROI production in the presence of infection thus overcoming endotoxin tolerance. In animals, LPS hyporesponsiveness is a highly effective mechanism of protection against the lethal syndrome of severe sepsis (21-23), but in humans, it is associated with increased mortality (24). Understanding endotoxin tolerance in neonates may assist in creating future adjuvant therapies for both sepsis and adverse outcomes, and optimizing $1,25 \mathrm{OHD}$ is a promising solution especially with rising antibiotic resistance (25).

\section{METHODS}

\section{Reagents and Antibodies}

The reagents and antibodies used in this study are PBS tablets (Oxoid, Thermo Fisher Scientific, Cambridge, UK); phycoerythrin labeled $\mathrm{CD} 11 \mathrm{~b}$ and BD FACS lysing solution (BD Biosciences, Oxford, UK); Alexa Fluor 647 anti-human toll-like receptor 4 (eBiosciences, Hatfield, UK); penicillin-streptomycin, liquid $100 \mathrm{ml}$ (GibcoBRL Life Technologies/Invitrogen, Dun Laoghaire, Ireland); L-glutamine (GibcoBRL Life Technologies/Invitrogen); Dulbecco's modified eagle medium (GibcoBRL Life Technologies/Invitrogen); phosphoAkt rabbit mAb Alexa Fluor 647 conjugate (Brennan and Company, Dublin, Ireland); phospho-Erk RABBIT mAb Alexa Fluor 488 conjugate(Brennan and Company); phospho p38 MAPK rabbit mAb and anti-rabbit IgG PE conjugate (Brennan and Company); TLR4 signaling inhibitor TAK-242 (InvivoGen, Toulouse, France); formaldehyde 16\% solution methanol free (Electron Microscopy Sciences, Hatfield, PA). All remaining reagents including 1,25-dihydroxyvitamin D3 were purchased from Sigma Aldrich, Dublin, Ireland unless otherwise stated.

\section{Study Population}

This study was approved by the ethics committees of National Maternity Hospital, Dublin, Ireland, for the study period from August 2011 to June 2014. All participants were informed of the nature of the study, and written consent was obtained prior to recruitment. The following patient groups were recruited:

1. Adult controls: Healthy hospital and laboratory adult staff aged 22-48 y, both males and females.

2. Term neonatal controls: Normal term neonates delivered by normal spontaneous vaginal delivery. All infants had an uncomplicated postnatal course with Apgar scores of 9 at $5 \mathrm{~min}$.

3. Preterm infants: <32-wk gestation admitted to neonatal intensive care unit within $24 \mathrm{~h}$ of birth. All preterm infants who participated in this translational study were delivered by spontaneous vaginal delivery. Infants $>32$-wk gestation, infants with major congenital anomalies, maternal substance abuse, postnatal transfers from other hospitals, and those who died prior to admission to the neonatal intensive care unit were excluded from the study.

All blood samples for in vitro studies were collected in accordance with established standard operating procedure in a closed system sodium citrate anticoagulated blood bottle and analyzed within 90 min of collection. The adult samples were used as an internal positive control in the experimental model. Blood samples $(1 \mathrm{ml})$ were taken from preterm infants during routine phlebotomy from either a central line or peripheral sample and from cord blood of normal term neonates born by vaginal delivery. Whole blood was incubated for $1 \mathrm{~h}$ at $37^{\circ} \mathrm{C}$ with the pro-inflammatory agent LPS $1 \mu \mathrm{g} / \mathrm{ml}$ to mimic an inflammatory response in vitro. In addition, $10^{-8} \mathrm{M}$ of $1,25 \mathrm{OHD}$ was added following a dose-response study (results not shown) (26). 


\section{Vitamin D Measurement}

All recruits had their baseline vitamin D status assessed. Blood samples $(1 \mathrm{ml})$ were collected in clotted specimen bottles, and the separated serum were stored at $-80^{\circ} \mathrm{C}$ freezer which were later sent in batches for analysis of $25 \mathrm{OHD}$ concentration using the Elecsys vitamin $\mathrm{D}$ total automated competitive binding protein assay (Roche Diagnostics GmbH, Mannheim, Germany) (27). This method employs a recombinant vitamin D binding protein (VDBP) as capture protein and has been standardized against LC-MS/MS, which in turn has been standardized to the NIST standard. The coefficients of variation for the $25 \mathrm{OHD}$ assay determined at assay validation were as follows: inter-assay coefficients of variation, $13.1 \%$ at a concentration of $14.7 \mathrm{nmol} / \mathrm{l}, 7.7 \%$ at $43.7 \mathrm{nmol} / \mathrm{l}$, and $2.2 \%$ at $110.9 \mathrm{nmol} / \mathrm{l}$; intra-assay coefficients of variation, $8.8 \%$ at a concentration of $14.7 \mathrm{nmol} / \mathrm{l}, 3.6 \%$ at $43.7 \mathrm{nmol} / \mathrm{l}$, and $2.1 \%$ at $110.9 \mathrm{nmol} / \mathrm{l}$.

\section{Quantification of Cell Surface Antigen Expression}

The expression of CD11b and TLR4 antigens on the surface of neutrophils and monocytes was measured by flow cytometry as previously described. Whole blood $(50 \mu \mathrm{l})$ was treated with $5 \mu \mathrm{l}$ of PE-CD11b and $2.5 \mu \mathrm{l}$ anti-human TLR4 antibody and left at $4{ }^{\circ} \mathrm{C}$ for $20 \mathrm{~min}$. The flow cytometer used was a BD Accuri C6 with a C Flow Plus software. A minimum of 5,000 events were collected and analyzed (28).

\section{Respiratory Burst Activity: Generation of ROIs}

Generation of ROIs was evaluated by flow cytometry using the technique of Smith and Weidemann (29). Whole blood $(50 \mu \mathrm{l})$ was incubated with DHR $123(50 \mu \mathrm{l})$ and PBS $(450 \mu \mathrm{l})$ at $37^{\circ} \mathrm{C}$ for $10 \mathrm{~min}$. Cells were stimulated with $1 \mu \mathrm{l}(16 \mu \mathrm{M})$ of PMA for $20 \mathrm{~min}$ at $37^{\circ} \mathrm{C}$. The reaction was then halted by placing samples on ice. Neutrophil and monocyte fluorescence intensity was assessed by flow cytometry and expressed as Ln mean channel fluorescence (LnMCF). DHR 123 has been shown to detect mainly intracellular $\mathrm{H}_{2} \mathrm{O}_{2}$ and $\mathrm{OH}$ radical production (29).

\section{Assessment of ROI Production with Specific NADPH Oxidase Inhibitor APO and Nonspecific NADPH Oxidase Inhibitor DPI} The inhibitors of NADPH oxidase system APO (highly specific) and DPI (nonspecific) were solubilized in 100\% dimethyl sulfoxide (DMSO). In our reconstitution of the NADPH oxidase inhibitors, both salts were diluted 100-fold with DMEM upon addition to whole blood thus exposing the blood to a final DMSO concentration of $1 \%$. Given the chance of DMSO toxicity and possibility of false-positive results as noted earlier, we employed a vehicle control using this concentration which had no effect on whole blood. We pretreated whole blood samples for $30 \mathrm{~min}$ with increasing concentrations of 1,10 , and $100 \mu \mathrm{M}$ for both inhibitors prior to addition of LPS $(1 \mu \mathrm{g} / \mathrm{ml})$ and $1,25 \mathrm{OHD}\left(10^{-8} \mathrm{M}\right)$.

\section{Inhibition of PI3K, P38, and ERK Effector Enzymes}

The effect of blocking NADPH oxidase effector enzymes on 1,25OHD whole blood neutrophil ROI response was assessed using the following: PI3K inhibitor (LY 294002), ERK inhibitor (PD 98059), and p38 inhibitor (SB 203580), at increasing concentrations of 1,10 , and 50 $\mu \mathrm{M}$ for $30 \mathrm{~min}$ of pre incubation. The salts were solubilized in $100 \%$ DMSO and further diluted 100-fold before addition to whole blood as previously explained.

\section{Assessment of TLR4 Inhibition}

Specific TLR4 blocker, TAK-242 (CLI-095), was solubilized in 100\% DMSO and diluted further 100 -fold as previously explained. Whole blood was pretreated and incubated with TAK-242 at increasing concentrations of 1,5 , and $10 \mu \mathrm{M}$ for 30 min prior to experiments as previously described (30).

\section{Assessment of Signaling Proteins; Phosphorylated Akt, p38, and ERK; and Effects of Inhibitors}

The action of $1,25 \mathrm{OHD}$ on the activation (phosphorylation) of neutrophil effector enzymes, Akt, p38, and ERK, by intracellular flow cytometry using term neonatal whole blood was assessed. The effects of blocking these enzymes with PI3K inhibitor LY 294002, P38 inhibitor SB 203580, and ERK inhibitor PD 98059 on phosphorylation of enzymes were also assessed. We used $10 \mu \mathrm{M}$ of each of these inhibitors which we had previously established to exert an effect. Methods for whole blood intracellular flow cytometry were adapted from Chow et al. (31). Hundred microliter of whole blood from healthy term infants was \pm pretreated and incubated with p38 MAPK inhibitor SB 203580, PI.3K inhibitor LY 294002, and ERK inhibitor PD 98059 for $30 \mathrm{~min}$. Whole blood was then pretreated with $\pm 10^{-8} \mathrm{M} 1,25 \mathrm{OHD}$ and incubated with \pm LPS $(1 \mu \mathrm{g} / \mathrm{ml})$ as in previous experiments. PMA was subsequently added and incubated for $20 \mathrm{~min}$ at $37^{\circ} \mathrm{C}$, rest of experiments as previously validated by Chow et al. (31).

For all the neutrophil function tests, the same person performed all the experiments which reduced variability of results. Each experiment on the same patient was always performed in duplicate, and any pair with a difference of more than $5 \%$ was discarded. The adult controls were recruited from the pediatric unit staffing and performed on the same day as the patient samples.

\section{Statistical Methods}

Matched paired $t$ tests were used to compare mean measurements between two independent groups. For comparing a cross group, one-way ANOVA with Tukey post hoc comparison method was used. Statistical analysis was carried out using ANOVA using PASW statistical package version 18. Significance was assumed for values of $P$ less than 0.05. Results are expressed as mean \pm SD unless otherwise indicated.

\section{STATEMENT OF FINANCIAL SUPPORT}

This research was funded by Children's Fund for Health, Children's University Hospital (Dublin, Ireland).

Disclosure: The authors declare no financial ties to the products used in the study and no potential or perceived conflict of interest.

\section{REFERENCES}

1. Levy O, Martin S, Eichenwald E, et al. Impaired innate immunity in the newborn: newborn neutrophils are deficient in bactericidal/permeabilityincreasing protein. Pediatrics 1999;104:1327-33.

2. Molloy EJ, O’Neill AJ, Grantham-Sloan JJ, Webb DW, Watson RW. Maternal and neonatal lipopolysaccharide and Fas responses are altered by antenatal risk factors for sepsis. Clin Exp Immunol 2008;151:244-50.

3. Gessler P, Nebe T, Birle A, Haas N, Kachel W. Neutrophil respiratory burst in term and preterm neonates without signs of infection and in those with increased levels of C-reactive protein. Pediatr Res 1996;39:843-8.

4. Carr R. Neutrophil production and function in newborn infants. Br J Haematol 2000;110:18-28.

5. Björkqvist M, Jurstrand M, Bodin L, Fredlund H, Schollin J. Defective neutrophil oxidative burst in preterm newborns on exposure to coagulasenegative staphylococci. Pediatr Res 2004;55:966-71.

6. Ambruso DR, Stork LC, Gibson BE, Thurman GW. Increased activity of the respiratory burst in cord blood neutrophils: kinetics of the NADPH oxidase enzyme system in subcellular fractions. Pediatr Res 1987;21: 205-10.

7. Barbour AG, Allred CD, Solberg CO, Hill HR. Chemiluminescence by polymorphonuclear leukocytes from patients with active bacterial infection. J Infect Dis 1980;141:14-26.

8. Romero R, Chaiworapongsa T. Preterm labor, intrauterine infection, and the fetal inflammatory response syndrome. Neoreviews 2002;3:73e-85.

9. Lu YC, Yeh WC, Ohashi PS. LPS/TLR4 signal transduction pathway. Cytokine 2008;42:145-51.

10. O'Hare FM, William Watson R, Molloy EJ. Toll-like receptors in neonatal sepsis. Acta Paediatr 2013;102:572-8.

11. Molloy EJ, O'Neill AJ, Doyle BT, et al. Effects of heat shock and hypoxia on neonatal neutrophil lipopolysaccharide responses: altered apoptosis, Tolllike receptor- 4 and CD11b expression compared with adults. Biol Neonate 2006;90:34-9.

12. Hmama Z, Nandan D, Sly L, Knutson KL, Herrera-Velit P, Reiner NE. 1alpha,25-dihydroxyvitamin $\mathrm{D}(3)$-induced myeloid cell differentiation is regulated by a vitamin $\mathrm{D}$ receptor-phosphatidylinositol 3-kinase signaling complex. J Exp Med 1999;190:1583-94.

13. Sly LM, Lopez M, Nauseef WM, Reiner NE. 1alpha,25-Dihydroxyvitamin D3-induced monocyte antimycobacterial activity is regulated by phospha- 
tidylinositol 3-kinase and mediated by the NADPH-dependent phagocyte oxidase. J Biol Chem 2001;276:35482-93.

14. Yang KD, Chen MZ, Teng RJ, et al. A model to study antioxidant regulation of endotoxemia-modulated neonatal granulopoiesis and granulocyte apoptosis. Pediatr Res 2000;48:829-34.

15. Barragán $M$, de Frias $M$, Iglesias-Serret $D$, et al. Regulation of Akt/PKB by phosphatidylinositol 3-kinase-dependent and -independent pathways in B-cell chronic lymphocytic leukemia cells: role of protein kinase C $\{$ beta $\}$. J Leukoc Biol 2006;80:1473-9.

16. Lai Y, Wang C, Hua Y, Hung C. Expression of neutrophil CD11b in preterm neonate and neonatal sepsis: a preliminary report. J Med Sci 2005;25:181-4.

17. Rebuck N, Gibson A, Finn A. Neutrophil adhesion molecules in term and premature infants: normal or enhanced leucocyte integrins but defective L-selectin expression and shedding. Clin Exp Immunol 1995;101:183-9.

18. Schildberger A, Rossmanith E, Eichhorn T, Strassl K, Weber V. Monocytes, peripheral blood mononuclear cells, and THP-1 cells exhibit different cytokine expression patterns following stimulation with lipopolysaccharide. Mediators Inflamm 2013;2013:697972.

19. Watson F, Robinson JJ, Edwards SW. Neutrophil function in whole blood and after purification: changes in receptor expression, oxidase activity and responsiveness to cytokines. Biosci Rep 1992;12:123-33.

20. Sadeghi K, Wessner B, Laggner U, et al. Vitamin D3 down-regulates monocyte TLR expression and triggers hyporesponsiveness to pathogen-associated molecular patterns. Eur J Immunol 2006;36:361-70.

21. Lehner MD, Ittner J, Bundschuh DS, van Rooijen N, Wendel A, Hartung T. Improved innate immunity of endotoxin-tolerant mice increases resistance to Salmonella enterica serovar typhimurium infection despite attenuated cytokine response. Infect Immun 2001;69:463-71.
22. Rayhane N, Fitting C, Lortholary O, Dromer F, Cavaillon JM. Administration of endotoxin associated with lipopolysaccharide tolerance protects mice against fungal infection. Infect Immun 2000;68:3748-53.

23. Yoza B, LaRue K, McCall C. Molecular mechanisms responsible for endotoxin tolerance. Prog Clin Biol Res 1998;397:209-15.

24. Cavaillon JM, Adib-Conquy M. Bench-to-bedside review: endotoxin tolerance as a model of leukocyte reprogramming in sepsis. Crit Care 2006;10:233.

25. Watts G. UK declares war on antimicrobial resistance. Lancet 2014;384:391.

26. Walker VP, Zhang X, Rastegar I, et al. Cord blood vitamin D status impacts innate immune responses. J Clin Endocrinol Metab 2011;96:1835-43.

27. van den Ouweland JM, Beijers AM, van Daal H, Elisen MG, Steen G, Wielders JP. Evaluation of 3-epi-25-hydroxyvitamin D3 cross-reactivity in the Roche Elecsys Vitamin D Total protein binding assay. Clin Chem Lab Med 2014;52:373-80.

28. Molloy EJ, O’neill AJ, Grantham JJ, et al. Labor induces a maternal inflammatory response syndrome. Am J Obstet Gynecol 2004;190:448-55.

29. Smith JA, Weidemann MJ. Further characterization of the neutrophil oxidative burst by flow cytometry. J Immunol Methods 1993;162:261-8.

30. Ii M, Matsunaga N, Hazeki K, et al. A novel cyclohexene derivative, ethyl (6R)-6-[N-(2-Chloro-4-fluorophenyl)sulfamoyl]cyclohex-1-ene-1carboxylate (TAK-242), selectively inhibits toll-like receptor 4-mediated cytokine production through suppression of intracellular signaling. Mol Pharmacol 2006;69:1288-95.

31. Chow S, Hedley D, Grom P, Magari R, Jacobberger JW, Shankey TV. Whole blood fixation and permeabilization protocol with red blood cell lysis for flow cytometry of intracellular phosphorylated epitopes in leukocyte subpopulations. Cytometry A 2005;67:4-17. 